\title{
Spin duality in the nucleon: Measurements at Jefferson Lab Hall A
}

\author{
Nilanga Liyanage ${ }^{1}$ \\ University of Virginia, Charlottesville, VA, USA
}

\begin{abstract}
The Bloom-Gilman duality has been experimentally demonstrated for spin independent structure functions. Duality is observed when the smooth scaling curve at high momentum transfer is an average over the resonance bumps at lower momentum transfer, but at the same value of scaling variable $x$. Signs of quark-hadron duality for the spin Dependant structure function $g_{1}$ of the proton has been recently reported by the Hermes collaboration and the Jefferson lab Hall B collaboration. Jefferson Lab experiment 01-012 used the polarized ${ }^{3} \mathrm{He}$ target in Hall A for a precision extraction of the neutron spin structure function $g_{1}^{n}$ and the virtual photon asymmetry $A_{1}^{n}$ in the resonance region up to $Q^{2}=4(\mathrm{GeV} / \mathrm{c})^{2}$. Data from E01-012 combined with Deep-Inelastic-Scattering data will provide a precision test of quark-hadron duality predictions for neutron spin structure functions. This will be one of the first precision tests of spin and flavor dependence of quark-hadron duality. The demonstration of duality for spin structure functions will enable the use of resonance data to study the nucleon spin structure in the very high $x$ region.
\end{abstract}

Thirty years ago Bloom and Gilman [1] made the observation that the scaling curve seen at high momentum transfer is an accurate average over the resonance bumps at lower momentum transfer but at the same value of $x$. This duality between the resonance region, which is best described by constituent quark models, and the scaling region, which is well described by $\mathrm{pQCD}$, hints a common origin for both regions. Several years after the observation of duality, De Rujula, Georgi and Politzer [2] suggested a framework based on the QCD operator product expansion (OPE) within which the averaging of the resonance bumps to the scaling curve can be interpreted in terms of the role of higher twists in DIS. In the OPE, the contributions to the QCD moments of the structure functions are organized according to the powers of $1 / Q^{2}$. The leading terms are associated with free quark scattering and are responsible for the scaling of the structure functions. The $1 / Q^{2}$ terms (higher twist terms) involve interactions between quarks and gluons and hence reflect elements of confinement dynamics. The experimental observation of duality for the unpolarized structure function $F_{2}$ of the proton indicates that the higher twist contributions to the proton $F_{2}$ moments are small or canceling on average, even in the low $Q^{2}$ regime where they should be maximized due to the $1 / Q^{2}$ behavior. Recent data from Jefferson Lab Hall C [3] have further confirmed that Bloom-Gilman duality holds to a few percent level down to small values of $Q^{2}$. The striking agreement shown by these data for unpolarized structure functions between the resonance and the scaling regions raises the question whether duality holds for polarized

${ }^{1}$ This contribution was presented by J.-P.Chen 


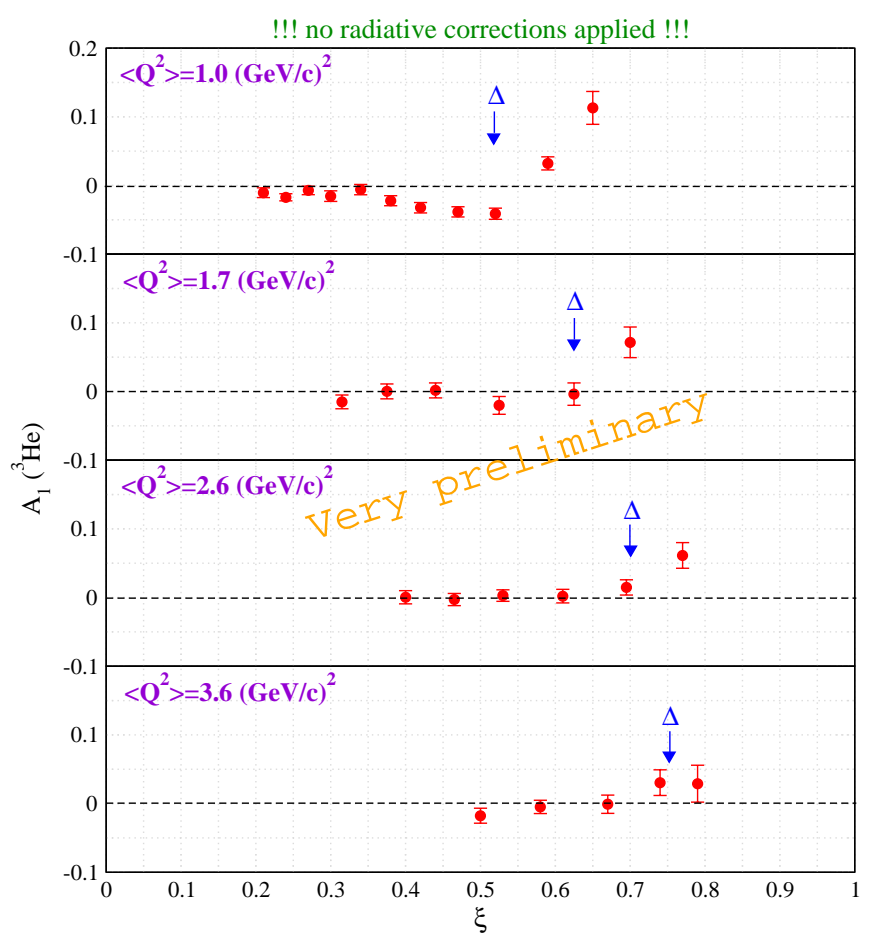

FIGURE 1. Preliminary results from E01-012 for $A_{1}^{3} \mathrm{He}$. No radiative corrections have been applied yet and the error bars are statistical only.

structure functions as well. Hermes collaboration has recently reported observing global quark-hadron duality for the spin structure function $g_{1}$ of the proton [4]. Data from Jefferson Lab Hall B collaboration show the onset of quark hadron duality for $g_{1}$ of the proton and the deuteron $[5,6]$. It should be noted that since $g_{1}$ of deuteron in the valence quark region is dominated by $g_{1}$ of the proton, spin duality observed in deuteron does not necessarily indicate spin duality for the neutron.

Jefferson Lab Hall A experiment E01-012 [7] used the Hall A polarized ${ }^{3} \mathrm{He}$ target for a precision extraction of the neutron spin structure function $g_{1}^{n}$ and the virtual photon asymmetry $A_{1}^{n}$ in the resonance region over a moderate $Q^{2}$ range (up to $Q^{2}=$ $\left.4(\mathrm{GeV} / \mathrm{c})^{2}\right)$. The results from this experiment, combined with Deep-Inelastic-Scattering data, will provide a precision test of quark-hadron (Bloom-Gilman) duality predictions for $g_{1}^{n}$ and $A_{1}^{n}$.

E01-012 ran in January and February, 2003. We used the polarized beam and the polarized ${ }^{3} \mathrm{He}$ target to measure the inclusive ${ }^{3} \vec{H} e\left(e, e^{\prime}\right) X$ reaction. Both Hall A High Resolution spectrometers (HRS) were used in a symmetric configuration in electron detection mode. Three beam energies, $3 \mathrm{GeV}, 4 \mathrm{GeV}$ and $5 \mathrm{GeV}$ were used with spectrometer angles of $25^{\circ}$ and $32^{\circ}$. At each kinematic setting cross sections and asymmetries 
were measured with the target spin parallel and perpendicular to the electron beam respectively.

We have formed raw asymmetries at each setting. We are currently extracting absolute cross sections to calculate $A_{1}$ and $g_{1}$ directly from our data. In the first pass analysis however, $A_{1}^{3} \mathrm{He}$ was calculated from the measured asymmetries assuming a constant value of 0.18 for the longitudinal and transverse cross section ratio $R$. No radiative corrections have been applied yet and the error bars are statistical only. Figure 1 shows $A_{1}^{3} \mathrm{He}$ at the four $Q^{2}$ values as a function of the the Nachtmann scaling variable $\xi=$ $2 x /\left(1+\sqrt{1+4 M^{2} x^{2} / Q^{2}}\right)^{2}$. The position of the $\Delta(1232)$ resonance is indicated by an arrow.

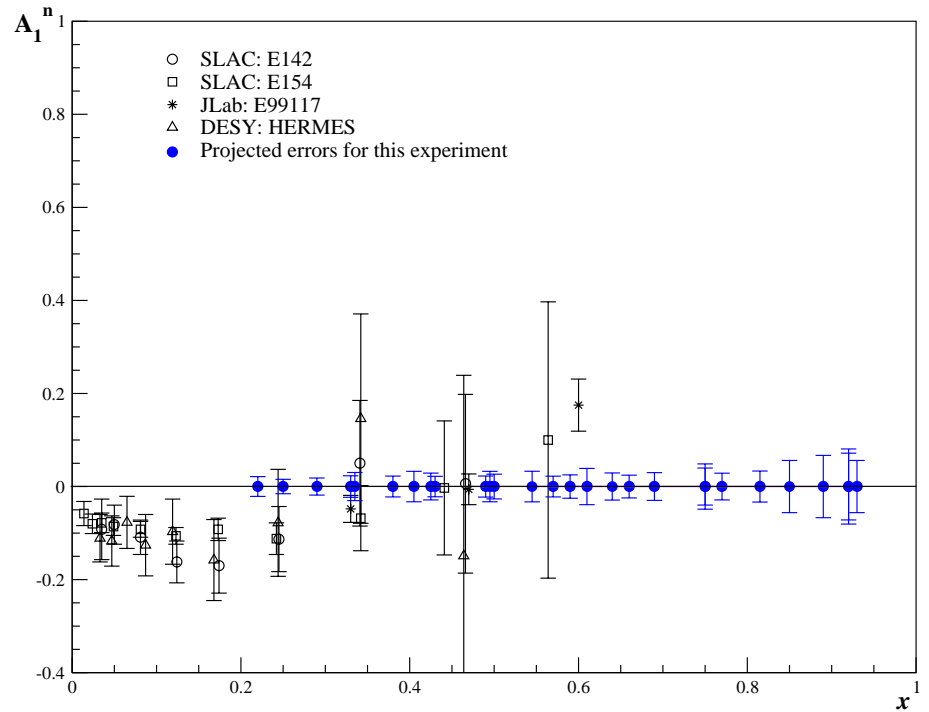

FIGURE 2. Available $A_{1}^{n}$ DIS data and projected E01012 error bars.

The most noticeable feature of the plots is the negative contribution due to $\Delta(1232)$ at low $Q^{2}$. It has been noted that quark-hadron duality for spin structure functions is not expected in the Delta resonance region at this low $Q^{2}$. Our data seem to indicate that as the non-resonant background is starting to dominate the delta region with increasing $Q^{2}, A_{1}$ goes from negative to positive. Recent results from Jefferson Lab Experiment 99-117 [8] indicate that $A_{1}^{n}$ in the DIS region also becomes positive at high $x$ as expected by relativistic constituent quark models and by pQCD inspired models. Figure 2 shows the $x$ range covered by E01-012 data, compared to the $x$ range currently covered by the DIS data. As the plot indicates for $0.2<x<0.63$ duality can be tested with the current data. With the Jefferson Lab $12 \mathrm{GeV}$ upgrade, the precision and the kinematic coverage of $A_{1}$ data in both DIS and resonance regions will improve significantly. This will enable

2 The Nachtmann scaling variable takes into account the target mass corrections which become important at low values of $Q^{2}$. Note that for high $Q^{2}, \xi \rightarrow x$. 


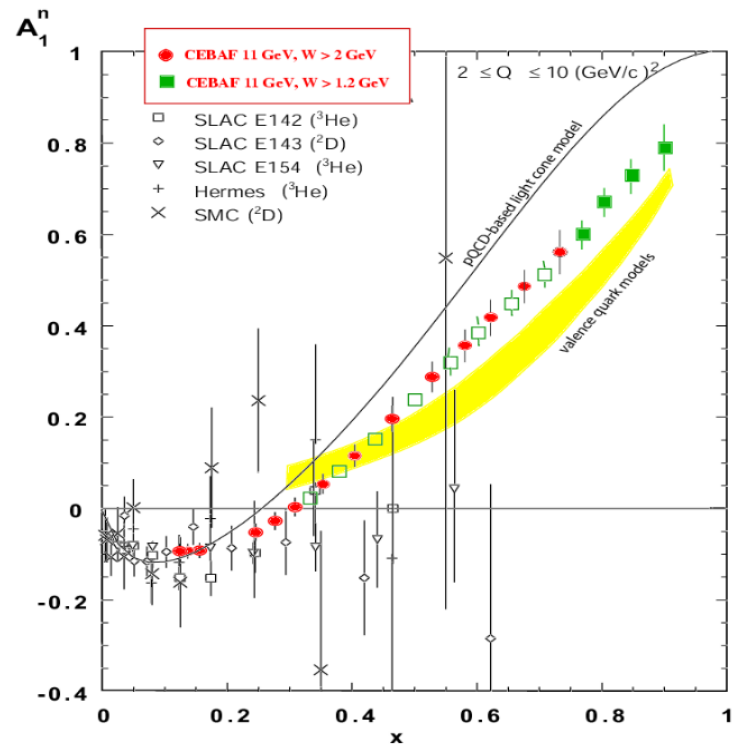

FIGURE 3. Projected $A_{1}^{n}$ data in IS and resonance regions with Jefferson lab $12 \mathrm{GeV}$ beam using the Hall A polarized ${ }^{3} \mathrm{He}$ target

us to extend the test of quark-hadron duality for spin structure function up to $x<0.75$.

While it is interesting in its own right to test quark-hadron duality for spin structure functions, such a test can lead to very important applications. If duality is established between the resonance region and the DIS region in the overlap range of $x<0.75$, we may be able to use the resonance data to study polarized quark distribution functions up to very high values of $x$. Figure 3 shows the projected $x$ coverage for DIS and resonance regions with Jefferson lab $12 \mathrm{GeV}$ beam.

\section{REFERENCES}

1. E. D. Bloom and F. J. Gilman, Phys. Rev. Lett. 25, 1140 (1970); Phys. Rev. D 4, 2901 (1971).

2. De Rujula, H Georgi, and H. D. Politzer, Ann. Phys. (N.Y.) 103 315(1977).

3. I. Niculescu et al., Phys. Rev. Lett. 85, 1186 (2000); I. Niculescu et al., Phys. Rev. Lett. 85, 1182 (2000).

4. A. Airapetian et al., Phys. Rev. Lett. 90, 092002 (2003).

5. Vipuli Dharmawardane, Ph.D Thesis, Old Dominion University (2004)

6. Yelena Prok, Ph.D Thesis, University of Virginia (2004).

7. Jefferson Lab Experiment E01-012, J.P Chen, S. Choi and N. Liyanage spokespeople.

8. X. Zheng et al. Phys. Rev. Lett. 92, 012004 (2004) [arXiv:nucl-ex/0308011]. 